\title{
Estatus de selenio en equinos Criollo-Chileno a pastoreo y su respuesta a la suplementación
}

\section{Selenium metabolic status and response to supplementation in grazing Chilean-Criollo horses}

\author{
Macarena Rioseco H, ${ }^{1}$ M.Sc, Mirela Noro, ${ }^{1,2 *}$ Ph.D, Ricardo Chihuailaf $\mathrm{V}^{3}{ }^{3} \mathrm{Ph} . \mathrm{D}$, Fernando \\ Wittwer M, ${ }^{1}$ M.Sc.
}

\begin{abstract}
${ }^{1}$ Universidad Austral de Chile, UACh, Instituto Ciencias Clínicas Veterinarias, Casilla 567, Valdivia, Chile. ${ }^{2}$ Universidade Federal do Pampa, UNIPAMPA, Curso de Medicina Veterinária, Uruguaiana, Brasil. ${ }^{3}$ Universidad Católica de Temuco, Escuela de Medicina Veterinaria, Temuco, Chile. *Correspondencia: mirelanoro@gmail.com
\end{abstract}

Recibido: Febrero de 2013; Aceptado: Agosto de 2013.

\section{RESUMEN}

Objetivo. Dos experimentos se realizaron para determinar el estatus de selenio en equinos CriolloChileno a pastoreo y, evaluar la respuesta a la suplementación con $\mathrm{Na}_{2} \mathrm{SeO}_{3}$. Materiales y métodos. Exp.1 A caballos pertenecientes a 10 criaderos del sur de Chile se les determinó la actividad sanguínea de glutatión peroxidasa (GPx, EC.1.11.1.9) (AcGPx). Los animales pertenecieron a 3 grupos, pastoreo de otoño ( $P O, n=40)$, pastoreo de otoño y suplementados con avena $(P O+A, n=47)$, y, pastoreo de primavera (PP, $n=41)$. Exp2Se utilizaron equinos con carencia de selenio, distribuidos en tres grupos: $\mathrm{G} 1, \mathrm{n}=7$, tratado con $\mathrm{Na}_{2} \mathrm{SeO}_{3}$ el día 0 ( $\mathrm{Se}=0.05 \mathrm{mg} / \mathrm{kg} \mathrm{pv}, \mathrm{im}$ ); G2, $\mathrm{n}=8$, suplementado con $\mathrm{Na}_{2} \mathrm{SeO}_{3}$, los días 0 y 15 y GC, $n=8$, control. La AcGPx se determinó al día 0,30 y 120 . Resultados. La medianá (Me) de la AcGPx en todos los grupos fue menor al límite adecuado ( $>130 \mathrm{U} / \mathrm{gHb}$ ), mayor en $\mathrm{PO}+\mathrm{A}$ $\left(M_{e}=92, P_{25 \%}=47, P_{75 \%}=129 \mathrm{U} / g \mathrm{Hb}\right)$ y $P P\left(M_{e}=85, P_{25 \%}=45, P_{75 \%}=114 \mathrm{U} / g H b\right)$ que en $P O\left(M_{e}=35\right.$, $\left.\mathrm{P}_{25 \%}=20, \mathrm{P}_{75 \%}=85 \mathrm{U} / \mathrm{gHb}\right)(\mathrm{p}<0.05)$. En el experimento 2 la AcGPX fue similar y menor al adecuado en todos los grupos. La suplementación con $\mathrm{Na}_{2} \mathrm{SeO}_{3}$ aumentó $(p<0.05)$ la AcGPx en $\mathrm{G} 1(121 \pm 52 \mathrm{U} / \mathrm{gHb})$

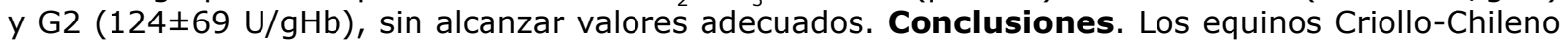
a pastoreo en el sur de Chile presentan carencia de Se, mayor en otoño que primavera y menor al suplementar con avena. Adicionalmente, la administración parenteral de $\mathrm{Na}_{2} \mathrm{SeO}_{3}$ mejora el estatus de Se, pero sin revertir el cuadro carencial.

Palabras clave: Equinos, GPx, selenito de sodio, variaciones estacionales (Fuente: MeSH).

\section{ABSTRACT}

Objective. Two experiments were conducted to determine the selenium status in grazing ChileanCriollo horses and evaluate their response to $\mathrm{Na}_{2} \mathrm{SeO}_{3}$ supplement. Material and methods. Exp.1The blood activity of glutathione peroxidase (GPx, EC.1.11.1.9) (AcGPx) was determined in horses kept grazing in 10 breeding farms from the south of Chile: $P O, n=40$ horses were kept grazing during autumn (PO), 47 horses were kept grazing during autumn and supplemented with oat grain $(P O+A)$, and 23 horses were kept grazing during spring (PP). Exp.223 horses kept on pasture were allotted 
into three groups: $\mathrm{G} 1, \mathrm{n}=7$, supplemented with a dose of $\mathrm{Na}_{2} \mathrm{SeO}_{3}(\mathrm{Se}=0.05 \mathrm{mg} / \mathrm{kg} \mathrm{bw}$, im) on day $0 ; \mathrm{G} 2, \mathrm{n}=8$, supplemented with similar doses of $\mathrm{Na}_{2} \mathrm{SeO}_{3}$, on days 0 and 15 ; and $\mathrm{GC}, \mathrm{n}=8$, control. AcGPx was determined on days 0,30 and 120. Results. Median (Me) of the AcGPx on all the farms were below the adequate limit ( $>130 \mathrm{U} / \mathrm{g} \mathrm{Hb})$, being higher in $\mathrm{PO}+\mathrm{A}\left(\mathrm{Me}=92, \mathrm{P}_{25 \%}=47, \mathrm{P}_{75 \%}=129\right.$ $\mathrm{U} / \mathrm{g} \mathrm{Hb})$ and $\mathrm{PP}\left(\mathrm{Me}=85, \mathrm{P}_{25 \%}=45, \mathrm{P}_{75 \%}=114 \mathrm{U} / \mathrm{g} \mathrm{Hb}\right)$ than $\mathrm{PO}\left(\mathrm{Me}=35, \mathrm{P}_{25 \%}=20, \mathrm{P}_{75 \%}=85 \mathrm{U} / \mathrm{g}\right.$ $\mathrm{Hb})(p<0.05)$. Initial AcGPx was similar and below adequate in the three groups and increased after $\mathrm{Na}_{2} \mathrm{SeO}_{3}$ administration $(\mathrm{p}<0.05)$ in $\mathrm{G} 1(121 \pm 52 \mathrm{U} / \mathrm{g} \mathrm{Hb})$ and $\mathrm{G} 2(124 \pm 69 \mathrm{U} / \mathrm{g} \mathrm{Hb})$. Conclusions. A deficiency is observed in Chilean-Criollo horses grazing during autumn and spring in southern Chile, parenteral administration of $\mathrm{Na}_{2} \mathrm{SeO}_{3}(0.05 \mathrm{mg} / \mathrm{kg}$ bw) in single or double doses improves the Se status without achieving adequate values.

Key words: Equine, GPx, sodium selenite, seasonal variations (Source: MeSH).

\section{INTRODUCCIÓN}

El selenio (Se) ejerce diversas funciones en el organismo formando parte de la estructura de diferentes selenoproteínas. Entre ellas, la enzima glutatión peroxidasa (GPx, EC 1.11.1.9) contiene en su estructura molecular 4 átomos de Se (1), cumpliendo un rol metabólico como antioxidante. La GPx se relaciona funcionalmente con la vitamina $E$ ya que ambas participan en la defensa de la célula contra el daño oxidativo producidos por los metabolitos reactivos de oxígeno (2).

El Se está ampliamente distribuido en el ambiente terrestre, pero su contenido y biodisponibilidad varía en el suelo y en los forrajes. Muchos factores afectan la concentración edáfica de este elemento como son la pluviosidad, época del año, irrigación y composición química del suelo (2). De estos factores depende indirectamente, además, la concentración foliar, sumado a otros como la especie forrajera, ya que las leguminosas acumulan menor cantidad que las gramíneas (2).

En el equino la correlación entre la actividad sanguínea de GPx y la concentración de Se en sangre es elevada $(r=0.84-0.94, p<0.01)$, por lo que la determinación de esta enzima permite estimar el estatus de Se en el organismo $(3,4)$. Por otra parte, la carencia de Se genera una disminución de la actividad de la GPx, favoreciendo el estrés oxidativo por un desequilibrio en el balance entre oxidantes $y$ antioxidantes (2).

En equinos de Sudamérica, incluyendo Chile, se han descrito enfermedades asociadas a la carencia de Se y/o vitamina E, entre las cuales están la miodegeneración nutricional, enfermedad degenerativa no inflamatoria que afecta la musculatura esquelética y cardiaca y la esteatosis o enfermedad de la grasa amarilla $(2,5)$.

El requerimiento mínimo de Se en el forraje para equinos es de $0.1 \mathrm{ppm}(2,6)$. En el sur de Chile se ha descrito que un $60 \%$ de los forrajes presenta concentraciones menores a $0.1 \mathrm{ppm}$
(7), considerado como valor mínimo aceptable para equinos, y consecuentemente insuficiente para satisfacer sus requerimientos nutricionales en entrenamiento, gestación o lactación. Debido a esto, tanto en Chile (5) como en otros países de Sudamérica (8), se han presentado cuadros clínicos asociados a la carencia de Se en rumiantes y equinos mantenidos en condiciones de pastoreo.

En equinos con miodegeneración asociada a carencia de Se, el uso de 1.1 a $1.7 \mathrm{mg}$ de Se como selenato de sodio, o selenito de sodio $\left(\mathrm{Na}_{2} \mathrm{SeO}_{3}\right)$ asociado o no con vitamina $\mathrm{E}(9)$, demostraron mejora clínica. La alimentación de equinos Criollo-Chileno solo en base a pastoreo con forrajes de bajo contenido de Se predispone a la presentación de carencias. Esta situación podría minimizarse con el uso estratégico de suplementos energéticos provenientes de otras regiones del país, como regiones del Bío-Bío y Metropolitana, en las cuales no se observan carencias de éste mineral, y consecuentemente los granos de cereales provenientes de estas regiones presentan adecuado aporte de Se. Por otro lado, la suplementación con Se sería una estrategia para controlar los estados carenciales en equinos a pastoreo (5).

El objetivo del estudio fue determinar el estatus metabólico de Se en otoño y primavera y evaluar el efecto de la suplementación con $\mathrm{Na}_{2} \mathrm{SeO}_{3}$ en equinos Criollo-Chileno mantenidos a pastoreo.

\section{MATERIAL Y MÉTODOS}

\section{Experimento 1.}

Ubicación y selección de los predios. Se seleccionaron en la Región de Los Ríos, Chile ( $39^{\circ}$ LS y $73^{\circ}$ LO), provincia de Valdivia, Chile, 10 criaderos de equinos Criollo-Chileno con un número mayor a 5 animales, que no utilizaban suplementación con Se, y mantenian los animales 
en pastoreo en praderas naturales fertilizadas durante las estaciones de otoño y primavera, con predominancia de Lolium spp. y un bajo contenido de Trifolium repens, Dactylis glomerata y Holcus lanatus. El clima de la región es tipo oceánico con influencia mediterránea húmeda, con una temperatura media anual de $12^{\circ} \mathrm{C}$ y una precipitación de $1.871 \mathrm{~mm}$ anual (10).

Animales y muestras. En un estudio dirigido se seleccionaron al azar equinos de 2 a 15 años de edad, de ambos sexos, clínicamente sanos, indistintamente al grado de ejercicio, y se establecieron 3 grupos: animales mantenidos sólo a pastoreo en otoño, grupo $\mathrm{PO}, \mathrm{n}=40$; animales mantenidos a pastoreo en otoño $y$ suplementados con avena en grano, grupo $\mathrm{PO}+\mathrm{A}$, $\mathrm{n}=41 ; \mathrm{y}$ por último, animales mantenidos a pastoreo en primavera, grupo PP, $n=47$. Se muestrearon entre 2 y 5 animales de cada criadero. De cada animal se obtuvo una muestra de sangre heparinizada de $5 \mathrm{~mL}$ mediante venopunción yugular.

\section{Experimento 2.}

Animales. Se selecionaron 24 equinos CriolloChileno de ambos sexos, entre 2 a 12 años de edad carentes de $\mathrm{Se}$, según sus valores de actividad sanguínea de la enzima GPx $(<130 \mathrm{U} / \mathrm{g}$ Hb) (Tabla 1).

Tabla 1. Clasificación de los animales en cuanto al estatus metabólico de Se según la actividad sanguínea de glutatión peroxidasa (GPX) (1).

\begin{tabular}{lc}
\hline \multicolumn{1}{c}{ Estatus } & Actividad sanguínea GPx (U/g Hb) \\
\hline Adecuado & $>130$ \\
Marginal & $101-130$ \\
Bajo & $60-100$ \\
Carente & $<60$ \\
\hline
\end{tabular}

Diseño experimental. Los animales se distribuyeron al azar en tres grupos: $G 1, n=7$, suplementado parenteralmente con $\mathrm{Na}_{2} \mathrm{SeO}_{3}$ (1.67\%, selenito de sodio p.a, Art. 107709, Merck Darmstadt, Alemania) en dosis única ( $\mathrm{Se}=0.05 \mathrm{mg} / \mathrm{kg} \mathrm{pv}$, im) el día 0 del experimento; $\mathrm{G} 2, \mathrm{n}=8$, suplementado con $\mathrm{Na}_{2} \mathrm{SeO}_{3}$ ( $\mathrm{Se}=0.05$ $\mathrm{mg} / \mathrm{kg} \mathrm{pv}, \mathrm{im}$ ) al día 0 y 15 del experimento y; $\mathrm{GC}, \mathrm{n}=8$, no suplementado, inyectado i.m. en el músculo glúteo medio con volumen equivalente de solución fisiológica como placebo el día 0 . El ensayo tuvo una duración de 120 días.

Todos los animales seleccionados fueron mantenidos a pastoreo en praderas naturales fertilizadas, con contenido de Se previamente determinado $(<0.04 \mathrm{ppm})$, sin uso de suplementos durante el periodo experimental. De cada animal se obtuvo una muestra de $5 \mathrm{~mL}$ de sangre heparinizada, mediante venopunción de la yugular, previo a la suplementación con Se (día 0) y posteriormente cada 30 días en cuatro oportunidades para determinar la actividad sanguínea de GPx y plasmática de aspartato aminotransferasa (AST, EC: 2.6.1.1).

Análisis. Las muestras se procesaron en el Laboratorio de Patología Clínica Veterinaria de la Universidad Austral de Chile, donde se determinó la concentración de hemoglobina mediante el método de la cianometahemoglobina en un fotocolorímetro HITACHI 4020 ${ }^{\circledR}$ (Japón). Luego, se preparó $1 \mathrm{~mL}$ de hemolizado conservándolo a $-25^{\circ} \mathrm{C}$ en microtubos de $1,5 \mathrm{~mL}$ para su análisis posterior. Además, se separó $1 \mathrm{~mL}$ de plasma que se almacenó a $-25^{\circ} \mathrm{C}$.

La actividad sanguínea de GPx se determinó mediante un kit Ransel, Randox $®$ y la actividad plasmática de AST por un kit Human ${ }^{\circledR}$, en un fotocolorímetro HITACHI $4020^{\circledR}$, en un lapso de tiempo no superior a 3 meses desde la obtención de la muestra.

Estadística. En ambos experimentos se determinó la normalidad y homocedasticidad de los datos mediante Shapiro-Wilk y Bartlett, respectivamente. En el experimento 1 se calculó la mediana $\left(M_{e}\right)$ y los percentiles $25 \%\left(P_{25 \%}\right)$ y $75 \%\left(P_{75 \%}\right)$. Las diferencias entre grupos se establecieron mediante la prueba de KruskalWallis. En el experimento 2 se analizaron los datos mediante un modelo lineal general de ANOVA de muestras repetidas: $Y_{i j}=\mu+S_{i}+M_{j}$ $+S^{*} M_{i j}+\varepsilon_{i j}$, donde: $Y_{i j}=$ variables dependientes, $\mu=$ media general, $S_{i}=$ efecto de la $i$-ésima suplementación, $M_{j}=$ efecto del $j$-ésima muestreo (días), $S^{*} M_{i j=}$ efecto de la interacción entre la i-ésima suplementación y j-ésima muestreo y, $\varepsilon_{i j}=$ residual del modelo. Se empleó un nivel de significancia de $p<0.05$, en el programa IBM SPSS 19.0 .

El estatus metabólico nutricional de Se fue establecido mediante los valores referenciales de la actividad de Se entregada por el kit (Ransel ${ }^{\circledR}$, Randox Laboratories Ltd., Crumlin, Irlanda del Norte) (1), siendo la presentación entre grupos constrastada por Chi-cuadrado o Fischer.

\section{RESULTADOS}

Experimento1. La totalidad de los criaderos presentó valores (Me) de la actividad de GPx bajo el límite de referencia adecuado (> $130 \mathrm{U} / \mathrm{g} \mathrm{Hb}$ ). La actividad de GPx fue inferior en el grupo PO que en $P O+A$ y PP $(p<0.05$, Tabla 2, Figura 1$)$. 
Tabla 2. Actividad sanguínea de glutatión peroxidasa (GPx, Ransel, Randox) en equinos CriolloChileno de criaderos del sur de Chile, mantenidos a pastoreo durante las estaciones de otoño (PO) y primavera (PP) y suplementados con avena en grano en otoño $(P O+A)$.

\begin{tabular}{lccc}
\hline & PO & PO+A & PP \\
\hline No criaderos & 10 & 10 & 9 \\
No equinos & 40 & 47 & 41 \\
GPx (U/g Hb) & & & \\
$\quad$ Mediana* & $35 \mathrm{a}$ & $92 \mathrm{~b}$ & $85 \mathrm{~b}$ \\
$\quad$ Percentil 25\% & 20 & 47 & 45 \\
$\quad$ Percentil 75\% & 85 & 129 & 114 \\
$\quad$ Valor mínimo & 12 & 13 & 14 \\
$\quad$ Valor máximo & 134 & 251 & 318 \\
\hline
\end{tabular}

$* \mathrm{p}=0.0002$

En el grupo PO sólo 2 de los animales ( $5 \%$ ) presentaron valores adecuados de GPx, porcentaje inferior $(p<0.05)$ a los grupos $P O+A$ ( $26 \%$ ) y PP $(17 \%)$. Consecuentemente, la mayor presentación de carencia de Se se observó en el grupo PO $(67.5 \%)(p<0.05)$ y la menor en los grupos $\mathrm{PO}+\mathrm{A}(31.9 \%)$ y PP $(34.1 \%)$, siendo estos últimos similares entre sí ( $p>0.05$, Figura 2$)$.

Experimento 2. Los valores de la actividad sanguínea de GPx previo a la suplementación

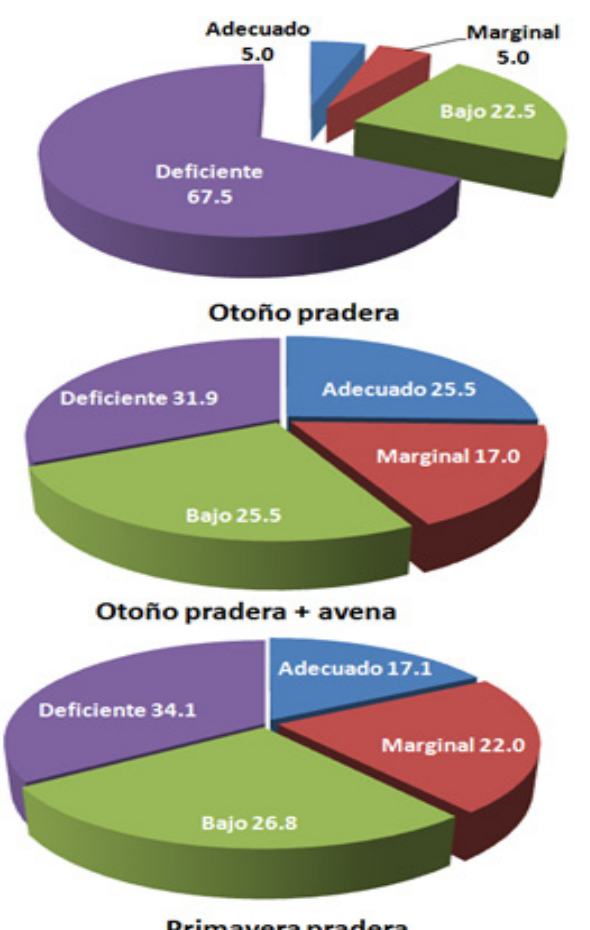

Figura 2. Distribución de equinos según su estatus de Se para grupos de animales en pastoreo de otoño, pastoreo de otoño y suplementados con avena y a pastoreo en primavera.

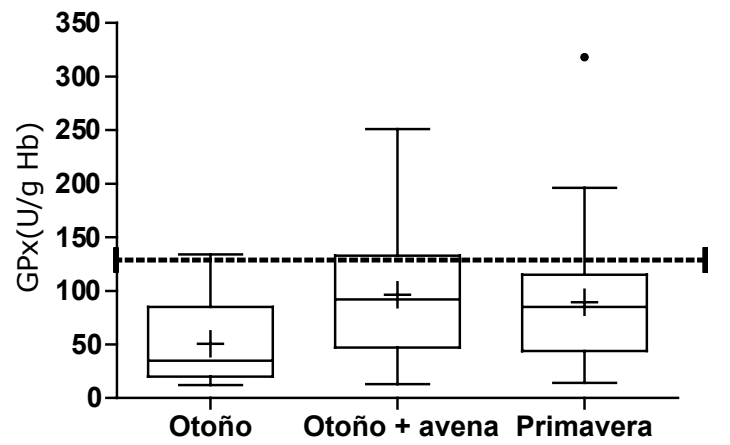

Figura 1. Gráfico de caja y bigotes de los valores de glutatión peroxidasa (GPx) en equinos en pastoreo de otoño, pastoreo de otoño y suplementados con avena y a pastoreo en primavera.----- indica límite para establecer estatus adecuado de Se (valores > $130 \mathrm{U} / \mathrm{g} \mathrm{Hb}$ ).

con $\mathrm{Na}_{2} \mathrm{SeO}_{3}$ se encontraron por debajo del valor considerado adecuado, y similares entre los tres grupos $(p<0.05$, Figura 3$)$. Posterior a la suplementación, se observó un aumento $(p<0.05)$ de la actividad sanguínea de GPx en forma paulatina en los grupos suplementados con $\mathrm{Na}_{2} \mathrm{SeO}_{3}$, sin diferencias entre ellos ( $\left.p>0.05\right)$. El grupo control también incrementó levemente su actividad de GPx a los 90 días del ensayo
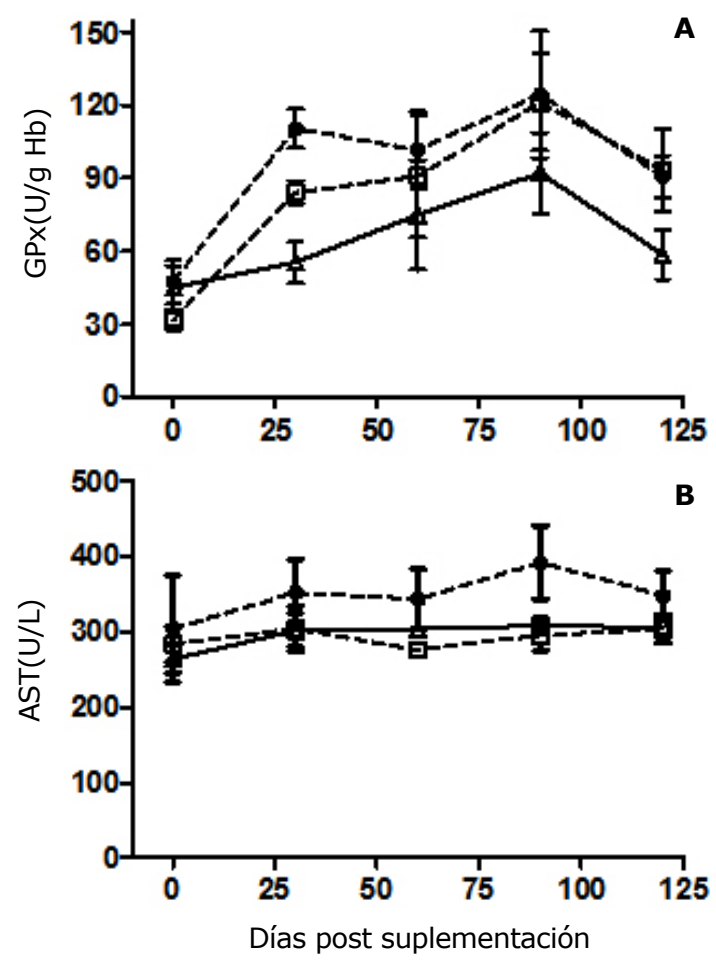

Figura 3. Actividad sanguínea de glutatión peroxidasa (GPx) y de aspartato aminotransferasa (AST) en equinos Criollo-Chileno, desde 0 a 120 días de una suplementación con selenito de sodio en dosis única $(\square)$ o repetida a los 15 días $(\bullet)$ y controles $(\Delta)$. 
$(p<0.05)$, momento en que los tres grupos presentaron sus mayores actividades de GPx, para luego disminuir a los 120 días (Figura 3).

El incremento en la actividad de las GPx posterior a la suplementación y, asociado al trascurso del ensayo en el grupo control, generó una reducción del número de caballos clasificados como carentes y bajos en Se, sin diferencias $(p>0.05)$ respecto a los que recibieron una o dos dosis de $\mathrm{Na}_{2} \mathrm{SeO}_{3}$; sin embargo la mayoría de los animales no logró alcanzar un estatus adecuado de Se durante el ensayo (Tabla 3).

Tabla 3. Distribución porcentual de caballos según su balance de selenio posterior a la suplementación con selenito de sodio en dosis única (G1) o repetida a los 15 días (G2) y controles (GC).

\begin{tabular}{lcccccc}
\hline \multirow{2}{*}{ Grupo } & \multirow{2}{*}{ Estatus } & \multicolumn{5}{c}{ Días } \\
\cline { 3 - 7 } Gosis única & Carente & 100 & 0 & 43 & 14 & 14 \\
& Bajo & 0 & 100 & 29 & 29 & 57 \\
& Marginal & 0 & 0 & 14 & 0 & 0 \\
& Adecuado & 0 & 0 & 14 & 57 & 29 \\
\hline G2 & Carente & 63 & 0 & 0 & 0 & 0 \\
Dosis repetida & Bajo & 37 & 25 & 50 & 63 & 88 \\
& Marginal & 0 & 63 & 38 & 12 & 0 \\
& Adecuado & 0 & 12 & 12 & 25 & 12 \\
\hline GC & Carente & 75 & 63 & 63 & 38 & 63 \\
Control & Bajo & 25 & 37 & 25 & 12 & 25 \\
& Marginal & 0 & 0 & 0 & 25 & 12 \\
& Adecuado & 0 & 0 & 12 & 25 & 0 \\
\hline
\end{tabular}

Por otro lado, los valores de la actividad plasmática de AST fueron similares $(p<0.05)$ y dentro del límite de referencia ( $<450 \mathrm{U} / \mathrm{L})$ en los tres grupos, permaneciendo constantes durante el período ( $p<0.05$, Figura 3$)$.

\section{DISCUSIÓN}

Experimento 1. La actividad sanguínea de GPx presentó variaciones entre los caballos en pastoreo de otoño y primavera, con valores inferiores en otoño en el que se observó un $95 \%$ de animales con valores carenciales. Esta diferencia no se registró entre los grupos de caballos en pastoreo de otoño suplementados con grano de avena con el grupo en pastoreo de primavera, los cuales presentaron mayor actividad de GPx y menor presentación de casos carenciales $(74.5 \%)$. Otro estudio realizado en Chile se observó $51.8 \%$ de animales con carencia de Se, con los menores valores de GPx observados en invierno (11). En República Checa se observó $48 \%$ de animales con carencia de Se (4). Por otro lado, al comparar con registros de rebaños de bovinos lecheros a pastoreo de la misma región del sur de Chile, se observa una mayor prevalencia de carencia de Se en los equinos. En los bovinos de la misma región del país se observó una prevalencia de $21.6 \%$, con menor actividad de GPx en invierno que en verano, sin observar diferencias entre la presentación de cuadros carenciales entre las otras estaciones del año (12). A su vez se debe considerar que la actividad de GPx refleja el estado metabólico nutricional de Se los tres meses anteriores al momento de realizar el análisis $(13,14)$.

La variación estacional en la presentación de desbalances carenciales de Se podría estar asociada a las diferencias en el aporte de Se en la dieta, producto del contenido mineral de la pradera y eventualmente de los suelos. La concentración foliar, así como la especie forrajera, afectan el contenido de Se de la pastura. Las leguminosas acumulan menor cantidad de Se que las gramíneas, si bien el Lolium sp., gramínea ampliamente utilizada en sur de Chile, presenta menor contenido de Se que la alfalfa o gramínea bermudas (2). Además existen diversos factores de riesgo para la carencia de Se y presentación de cuadros clínicos, como acidez del suelo, alto contenido de hierro y azufre, heno contaminado con hongos y dietas ricas en grasas no saturadas $(1,8)$.

La incorporación de otro alimento en la dieta de los equinos, como el grano de avena, sirvió como una fuente de Se capaz de incrementar la actividad sanguínea de GPx. Resultados similares fueron observados en yeguas gestantes suplementadas con una mezcla de granos, que presentaron una mayor concentración de Se plasmático comparado con aquellas mantenidas sólo a pastoreo (6). Se debe considerar que algunos caballos suplementados con avena en otoño presentaron valores de GPx similares a aquellos mantenidos sólo a pastoreo, situación que se podría atribuir a la variación en el contenido de Se de la avena. No obstante, este dato no pudo ser corroborado por el criadero, y no se pudo determinar el contenido de Se en los alimentos debido a dificultades analíticas.

El mayor crecimiento del forraje en primavera favorecería a la disminución en el contenido de Se en la planta, así como del mayor número de predios con contenidos deficientes de Se en sus forrajes en dicho período del año (1). Ahora si bien los equinos del presente estudio presentaron una alta prevalencia de carencia de Se, no se observaron cuadros clínicos. Las alteraciones asociadas a la carencia del Se en caballos varían con su magnitud y requerimiento para crecimiento o exigencias para trabajo o deporte. La enfermedad del músculo blanco o degeneración nutricional es una de las enfermedades clínicas que se observa como 
resultado de la carencia de Se en animales de granja, donde se incluye los caballos. La carencia moderada de Se puede llevar una diminución en la resistencia a las infecciones, infertilidad de yeguas y bajas tasas de crecimiento en los potrillos (2). En situaciones que requieren de una mayor actividad metabólica, como crecimiento, ejercicio, procesos inflamatorios y estrés se producen mayores demandas por oxígeno, generándose una gran cantidad de radicales libres, los cuales son nocivos al organismo. Cuando su producción supera las defensas antioxidantes, dentro de ellas la GPx, se producen lesiones tisulares, posibilitando la producción de tumores y enfermedades autoinmunes, lipoperoxidación lipídica, llevando a ruptura de las membranas celulares (5) y consecuentemente baja en la performance.

Experimento 2. La actividad sanguínea inicial media de GPx se encontró dentro de los valores considerados carentes en los caballos pertenecientes a los tres grupos ( $<60 \mathrm{U} / \mathrm{g} \mathrm{Hb})$ indicando un estado de carencia metabólico nutricional de Se, lo que estaría asociado a que su dieta se basaba sólo en el consumo de pradera con aporte de Se de 0.04 ppm, valor inferior al requerido en la dieta por la especie (0.1 ppm) (2).

El incremento leve y paulatino en la actividad sanguínea de GPx desde el día 30 posterior a la suplementación con $\mathrm{Na}_{2} \mathrm{SeO}_{3}$, que alcanzó el valor máximo al día 90 del experimento (Figura 2), no logró establecer un balance adecuado de Se. Considerando que la vida media de los eritrocitos en los equinos es de aproximadamente 155 días (13), y que la incorporación del Se ocurre durante la eritropoyesis, la estimación del estatus de Se en esta especie mediante la actividad sanguínea de GPx refleja el balance metabólico nutricional del mineral de 3 meses previo a la obtención de la muestra $(13,14)$.

Por otro lado, la mayoría de los productos comerciales en la forma inorgánica para suplementación de Se contienen $\mathrm{Na}_{2} \mathrm{SeO}_{3}$; sal que requiere de un largo y constante tiempo de administración, a pesar de lo cual no siempre se obtienen buenos resultados (15), observándose además efectos colaterales no deseados (16). Al respecto la administración de $\mathrm{Na}_{2} \mathrm{SeO}_{3}$ al 1.67 \% vía intramuscular no provocó reacciones sistémicas o locales en los animales en este experimento. Otro estudio con uso de selenato de bario $(0.5 \mathrm{mg} / \mathrm{kg} \mathrm{PV})$ de lenta liberación a animales con carencia de Se, logró incrementar la actividad de GPx eritrocitaria a los 60 días post tratamiento a valores adecuados. En este último estudio tampoco se observaron reacciones adversas en el punto de aplicación, salvo una leve molestia a la palpación y un aumento de temperatura local post tratamiento, situación que desapareció posterior al cuarto día post administración (5).

La actividad de la enzima AST se mantuvo sin variaciones durante todo el experimento y fue similar entre los grupos indicando posiblemente la ausencia de daño miocelular durante el período experimental. Además, se puede señalar que los animales analizados no evidenciaban signos clínicos de miopatía. La AST es un indicador de daño de células musculares al igual que la creatin quinasa (CK, EC: 2.7.3.2) por lo que su actividad plasmática es directamente proporcional a la magnitud del daño (17). Sin embargo por problemas analíticos no se pudo determinar la actividad de la CK en las muestras de los animales.

De acuerdo a lo anterior, sería necesario suplementar con Se no sólo los animales que constituyen los grupos de mayor riesgo sino que también en aquellas épocas del año en que la concentración del mineral es baja, situación que se deduce de una actividad de GPx disminuida.

Se concluye que la carencia de Se es frecuente en equinos a pastoreo en el sur de Chile, siendo esta mayor en otoño que primavera, situación que logra ser solo aminorada mediante la suplementación con avena en grano. La administración parenteral a caballos con carencia de $\mathrm{Se}$ de $\mathrm{Na}_{2} \mathrm{SeO}_{3}$ en dosis de $0.05 \mathrm{mg} /$ kg pv mejora el estatus de Se sin revertir la presentación del cuadro carencial.

\section{REFERENCIAS}

1. Wittwer F, Araneda P, Ceballos A, Contreras $\mathrm{PA}$, Andaur M, Böhmwald $\mathrm{H}$. Actividad de glutation peroxidasa (GSH-Px) en sangre de bovinos a pastoreo de la IX Región, Chile y su relación con la concentración de selenio en el forraje. Arch Med Vet 2002; 34:49-57.
2. Suttle NF. Mineral nutrition of livestock. 4th ed. UK: CAB International. 2010; 579.

3. Maylin GA, Rubin DS, Lein DH. Selenium and vitamin $\mathrm{E}$ in horses. Cornell Vet 1980; 70:272-289. 
4. Ludvikova E, Pavlata L, VyskoÄ il M, Jahn $P$. Selenium status of horses in the Czech Republic. Acta Veterinaria Brno 2005; 74:369-375.

5. Araya O, Urzua R, Bustamante H. Efecto del selenato de bario inyectable sobre la actividad de Glutation peroxidasa en caballos a pastoreo. Arch Med Vet 2004; 36:31-37.

6. Karren BJ, Thorson JF, Cavinder CA, Hammer CJ, Coverdale JA. Effect of selenium supplementation and plane of nutrition on mares and their foals: selenium concentrations and glutathione peroxidase. J Anim Sci 2010; 88:991-997.

7. Contreras PA, Wittwer $\mathrm{F}$, Matamoros $\mathrm{R}$, Mayorga IM, van Schaik G. Effect of grazing pasture with a low selenium content on the concentrations of triiodothyronine and thyroxine in serum, and GSH-Px activity in erythrocytes in cows in Chile. $\mathrm{N} Z$ Vet J 2005; 53: 77-80.

8. Cardona J, Reza L. Esteatosis en un burro (Equus asinus). Primer reporte en Colombia. Revista MVZ Córdoba 2011; 16:2793-2798.

9. NRC. Nutrient Requirements of Horses. 6th ed. Washigton D.C, USA: The National Academies Press. 2007.

10. Dirección Meteorológica de Chile Informe de precipitaciones. 2012. Acceso: 20 octubre 2012]. http://www.meteochile.cl/ precipitacion.html.
11. Tapia J, Menarim B, Stewart A, Noro M, Galecio JS. Variaciones estacionales y estado metabólico nutricional de Selenio en equinos del sur de Chile entre los años 2004 a 2011. Valdivia, Chile; 2012.

12. Wagemann CA, Wittwer $F$, Chihuailaf $\mathrm{RH}$, Noro M. Estudio retrospectivo de la prevalencia de desbalances minerales en grupos de vacas lecheras en el sur de Chile. Arch Med Vet 2014; in press.

13. Richardson SM, Siciliano PD, Engle TE, Larson CK, Ward TL. Effect of selenium supplementation and source on the selenium status of horses. J Anim Sci 2006; 84:1742-1748.

14. Calamari L, Abeni F, Bertin G. Metabolic and hematological profiles in mature horses supplemented with different selenium sources and doses. J Anim Sci 2010; 88:650-659.

15. Wichtel J], Grace ND, Firth EC. The effect of injectable barium selenate on the selenium status of horses on pasture. $\mathrm{N} \mathrm{Z}$ Vet J $1998 ; 46: 186-190$.

16. Moore RM, Kohn CW. Nutritional muscular dystrophy in foals. Compend. Cont Educ Pract Vet $1991 ; 13: 343-349$.

17. Kaneko JJ, Harvey JW, Bruss ML. Clinical Biochemistry of Domestic Animals. Sixth ed. San Diego: Academic Press. 2008. 